\title{
Identifying key genes and drug screening for preeclampsia based on gene expression profiles
}

\author{
ZHENGFANG XU ${ }^{1 *}$, CHENGJIANG WU $^{2 *}$, YANQIU LIU $^{1 *}$, NIAN WANG $^{1 *}$, SHUJUN GAO $^{3}$, \\ SHALI QIU ${ }^{3}$, ZHUTAO WANG ${ }^{3}$, JING DING $^{3}$, LUBIN ZHANG $^{3}$, HUI WANG $^{3}$, WEIJIANG WU ${ }^{3}$, \\ BING WAN ${ }^{4}$, JUN YU $^{1}$, JIE FANG $^{1}$, PEIFANG YANG $^{1}$ and QIXIANG SHAO ${ }^{3}$ \\ ${ }^{1}$ Department of Gynecology and Obstetrics, Affiliated Hospital of Jiangsu University, Zhenjiang, Jiangsu 212001; \\ ${ }^{2}$ Department of Clinical Laboratory, The Second Affiliated Hospital of Soochow University, Suzhou, Jiangsu 215000; \\ ${ }^{3}$ Reproductive Sciences Institute, Jiangsu Key Laboratory of Medical Science and Laboratory Medicine, \\ Department of Immunology, School of Medicine, Jiangsu University, Zhenjiang, Jiangsu 212013; \\ ${ }^{4}$ Department of Respiratory and Critical Care Medicine, The Affiliated Jiangning Hospital of Nanjing Medical \\ University, Nanjing, Jiangsu 210002, P.R. China
}

Received July 11, 2019; Accepted April 16, 2020

DOI: $10.3892 / 01.2020 .11721$

\begin{abstract}
Preeclampsia (PE) is characterized by gestational hypertension and proteinuria, and is a leading cause of maternal death and perinatal morbidity globally. Although the exact cause of PE remains unclear, several studies have suggested a role for abnormal expression of multiple genes. The aim of the present study was to identify key genes and related pathways, and to screen for drugs that regulate these genes for potential PE therapy. The GSE60438 dataset was acquired from the Gene Expression Omnibus database to analyze differentially expressed genes (DEGs). By constructing a protein-protein interaction network and performing reverse transcription-quantitative PCR verification, proteasome $26 \mathrm{~S}$ subunit, non-ATPase 14, prostaglandin E synthase 3 and ubiquinol-cytochrome $c$ reductase core protein 2 were identified as key genes in PE. In addition, PE was found to be associated with 'circadian rhythm', 'fatty acid metabolism', 'DNA damage response detection of DNA damage', 'regulation of DNA repair' and 'endothelial cell
\end{abstract}

Correspondence to: Professor Nian Wang, Department of Gynecology and Obstetrics, Affiliated Hospital of Jiangsu University, 438 Jiefang Road, Zhenjiang, Jiangsu 212001, P.R. China E-mail:2256659262@qq.com

Professor Qixiang Shao, Reproductive Sciences Institute, Jiangsu Key Laboratory of Medical Science and Laboratory Medicine, Department of Immunology, School of Medicine, Jiangsu University, 301 Xuefu Road, Zhenjiang, Jiangsu 212013, P.R. China

E-mail: shao_qx@ujs.edu.cn

${ }^{*}$ Contributed equally

Key words: preeclampsia, Gene Ontology biological process terms, Kyoto Encyclopedia of Genes and Genomes pathway, PSMD14, PTGES3, UQCRC2 development'. Through connectivity map analysis of DEGs, furosemide and droperidol were suggested to be therapeutic drugs that may target the hub genes for PE treatment. Results analysis of GSEA were included in the discussion section of this article. In conclusion, the current study identified novel key genes associated with the onset of PE and potential drugs for PE treatment.

\section{Introduction}

Preeclampsia (PE) occurs during pregnancy, and seriously affects the health of pregnant women and fetuses. PE is the leading cause of increased maternal and fetal mortality worldwide (1). The incidence rate of PE is $9.4 \%$ in China and $7-12 \%$ abroad (2). The pathogenesis of PE is complicated and has not yet been fully elucidated. In addition, PE is associated with multiple risk factors. Previous studies have demonstrated that PE is associated with diabetes (3), obesity (4) and hypertension (5). It is difficult to prevent and treat PE as there are currently no effective drugs available. PE shares several pathophysiological mechanisms with cardiovascular diseases (6), which suggests that statins may be useful in prevention and early management of PE. Nevertheless, clinical evidence for the effectiveness of statins in the treatment of PE is mainly limited to animal models (7). It is generally recognized that the main clinical manifestations of PE are hypertension and proteinuria; however, the organs of pregnant women and fetuses may be impaired to varying degrees, and the majority of pregnant women do not exhibit clinical symptoms until 20 weeks of gestation (8). Accumulating evidence has confirmed that the occurrence and development of PE are associated with multiple genes and cellular pathways; no single factor can explain the pathogenesis and mechanism of PE $(9,10)$. Due to the high mortality rates in PE, revealing the causes, underlying molecular mechanisms, identifying molecular biomarkers and exploring effective medicine for early diagnosis, prevention and personalized treatment, are extremely important. 
High-throughput sequencing and microarray technology play critical roles in revealing the pathogenesis of disease, and the structure and function of the genome (11). High-throughput analysis platforms combined with bioinformatics analysis can be used to discover key genes and search for effective drugs for therapy. Luo et al (12) identified 10 key genes in patients with PE compared with healthy donors. In addition, a study by Zhu et al (13) indicated that there were 11 upregulated microRNAs (miRNAs) and 23 downregulated miRNAs in patients with PE. However, the majority of previous studies focused on the significance of individual differentially expressed genes (DEGs) and did not investigate the association between the DEGs and PE as a whole. Therefore, previous studies may have missed biologically significant information about genes and their functions. In addition, few studies focused on screening of drugs for PE therapy.

In the present study, a gene expression profile dataset was obtained from the Gene Expression Omnibus (GEO) database (14). Gene set enrichment analysis (GSEA) was used to detect whether gene expression in defined functional groups is a common expression trend, which can link the microarray data with biological meaning (15). Furthermore, potential drugs for PE therapy were screened using connectivity map (CMAP) analysis. A number of studies have applied the CMAP database to disease treatment and drug development (16-18). The occurrence of PE is determined by changes in multiple genes. Therefore, the CMAP can indicate the structure of small molecule drugs for the treatment of PE. Recently, studies have focused on the role of gene expression and regulation in the development of PE (19-23). Therefore, the present study investigated novel key genes involved in the onset of PE. The aim of this study was to provide a novel perspective for further exploring the pathogenesis, treatment and prevention of PE.

\section{Materials and methods}

Data source. Transcriptome expression profiles of normotensive pregnant woman and patients with PE were obtained from the microarray dataset GSE60438 (24) from the GEO database (http://www.ncbi.nlm.nih.gov/geo/). The dataset was based on the GPL6884 platform (Illumina HumanWG-6 v3.0 Gene Expression BeadChip; Illumina, Inc.), which contains probe information, including cDNAs, oligonucleotides and open reading frames. The dataset consists of 48 human samples, including 23 normotensive samples and $25 \mathrm{PE}$ samples. The Bioconductor limma package (version 3.11) for R (version 4.0; http://www.bioconductor. org/packages/release/bioc/html/limma.html) was used to standardize, clean and convert the matrix data. Finally, the limma package was also used to identify DEGs (25). $\mathrm{P}<0.05$ and Ifold-changel $\geq 1.2$ were applied to screen DEGs in PE. The gplots package (version 3.0.3) was used to analyze the data and draw a heatmap plot (https://github. com/talgalili/gplots).

Functional annotation of DEGs. Functional annotation of DEGs consisted of Gene Ontology (GO) analysis of biological process (BP) terms (26) and Kyoto Encyclopedia of Genes and
Genomes (KEGG) pathway enrichment analysis (27). GO and KEGG pathway databases were extensively used in analyzing the BPs and cellular signaling pathways that DEGs were involved in. The Database for Annotation Visualization and Integrated Discovery online tool (version 6.8; https://david. ncifcrf.gov/) was used to perform analysis of GO BP terms and KEGG pathway enrichment of DEGs in PE (28). Significantly enriched pathways and GO-BP terms with $\mathrm{P}<0.01$ were focused on in the present study.

GSEA analysis of DEGs. GSEA is a tool for genome-wide expression profile data analysis that detects gene sets rather than individual gene expression changes. Therefore, GSEA could identify genes with small differences in expression and important functions, making the analysis more accurate and comprehensive (15). GSEA (version 2; http://software.broadinstitute.org/gsea/index.jsp) was used to determine whether an a priori defined set of genes enriched in GO BP terms and KEGG pathways shows significant differences between gene expression data of normotensive patients and patients with PE (29).

Protein-protein interaction (PPI) network analysis. STRING (version 11.0; https://string-db.org/) is a database for searching interactions of known and predicted proteins, which was used to study the molecular mechanism of disease and investigate novel drug targets. In the present study, a PPI network of DEGs was extracted using the STRING tool. The PPI network was constructed using Cytoscape (version 3.4.0; https://cytoscape.org/). Only experimentally validated PPIs with a combined score $>0.4$ were selected to construct the PPI network. Next, the hub genes associated with PE were identified in networks using CytoHubba (version 0.1; https://www.cytoscape.org) (30). CytoHubba provided a user-friendly interface to explore important nodes in biological networks. The Molecular Complex Detection (MCODE; version 1.4.2) (31) plugin in Cytoscape was then utilized to screen the top three modules in the PPI network. Parameters were set as follows: Degree cutoff, 2; node score cutoff, 0.2; k-core, 2; and maximum depth, 100.

CMAP analysis. CMAP (http://www.broadinstitute. org/cmap/) is a database of small molecule drugs, gene expression and disease-related biological applications (32). CMAP contains the gene expression profiles of breast cancer MCF7 cells, prostate carcinoma PC3 cells and acute myeloid leukemia HL60 cells treated with different drugs. The CMAP database contains 1,309 small molecule drugs and $>7,000$ gene expression profiles. Each small molecule drug treated different cells at different concentrations and time points. The gene expression profiles were analyzed to distinguish positive and negative regulatory gene groups. The score was based on the similarity of gene expression profiles between cells treated with small molecule drugs and disease gene expression profiles. Using CMAP analysis, gene expression profiles were compared to identify the drugs that were highly related to diseases, to deduce the main chemical structures of most drug molecules, and to summarize the possible mechanisms of drug molecules. Through CMAP analysis of DEGs in PE, small molecule 
Table I. Primer sequences of hub genes and the annealing temperature used for reverse transcription-quantitative PCR.

\begin{tabular}{|c|c|c|}
\hline Primer & Primer sequence $\left(5^{\prime}-3^{\prime}\right)$ & Annealing temperature $\left({ }^{\circ} \mathrm{C}\right)$ \\
\hline \multirow[t]{2}{*}{ PSMD14 } & F: CAGATGCTCCTGCAGTGGAC & 61.5 \\
\hline & R: AACAACCATCTCCGGCCTTC & \\
\hline \multirow[t]{2}{*}{ PTGES3 } & F: ACGTTCATTCTCCGTCCTCG & 56.7 \\
\hline & R: AAGCAGGCTGCATTGTGAAC & \\
\hline \multirow[t]{2}{*}{ UQCRC2 } & F: ACGCAAAAAGCAGTGACGTA & 60.3 \\
\hline & R: CCGGCTCTGGTTAGTAGCTT & \\
\hline \multirow[t]{2}{*}{ SUMO2 } & F: GCGGACCTGGTACCTCTTTT & 53.6 \\
\hline & R: TCCAACTGTGCAGGTGTGTC & \\
\hline \multirow[t]{2}{*}{ ACTR3 } & F: TTCTTGCTACTGCTTCGGCTT & 61.1 \\
\hline & R: AGCTTGATCACCCACTTTTGC & \\
\hline \multirow[t]{2}{*}{$\beta$-actin } & F: AGCGAGCATCCCCCAAAGTT & 56 \\
\hline & R: GGGCACGAAGGCTCATCATT & \\
\hline
\end{tabular}

drugs that could be used to treat PE were investigated. The cut-off value was defined as Iconnectivity scorel $>0.7$ to identify candidate small molecule drugs associated with PE.

Extraction of tissue RNA and detection of expression of key genes by reverse transcription-quantitative $(R T-q) P C R$. A total of 38 placenta specimens were collected between March 2018 and December 2018, including 28 specimens from patients with PE and 10 from healthy pregnant women at the Department of Gynecology and Obstetrics, Affiliated Hospital of Jiangsu University (Zhenjiang, China). The diagnosis of PE was given according to the guidelines for the diagnosis and treatment of hypertensive disorders in pregnancy (2015) from the Group of Hypertensive Diseases in Pregnancy, the Society of Obstetrics and Gynecology of the Chinese Medical Association (33). The patients with PE did not suffer from any other disease. The age range of patients with PE and healthy controls was 20-45 years old. The researchers obtained verbal informed consent before collecting patient samples. Written informed consent was waived by the Ethics Committee of the Affiliated Hospital of Jiangsu University. The experiment was approved by the Ethics Committee of the Affiliated Hospital of Jiangsu University (approval no. 20170415). For evaluation of the expression of key genes in the placenta of patients and healthy women, tissue RNA was extracted using TRIzol ${ }^{\circledR}$ (Invitrogen; Thermo Fisher Scientific, Inc.). Total RNA was reverse transcribed into cDNA using PrimeScript ${ }^{\mathrm{TM}}$ RT Master Mix (Takara Biotechnology Co., Ltd.) at $42^{\circ} \mathrm{C}$ for 30 min. qPCR was carried out using TB Green ${ }^{\mathrm{TM}}$ Fast qPCR mix on a CFX96 ${ }^{\text {TM }}$ Real-Time PCR Detection system (Bio-Rad Laboratories, Inc.). For the analysis of gene expression, quantification of relative mRNA levels was carried out using the $2^{-\Delta \Delta C q}$ method (34). Values were normalized to endogenous $\beta$-actin expression. Each experiment was independently repeated three times. All primers used for
RT-qPCR are listed in Table I. The thermocycling conditions were as follows: $95^{\circ} \mathrm{C}$ for $30 \mathrm{sec}$, followed by 40 cycles of $95^{\circ} \mathrm{C}$ for $5 \mathrm{sec}$ and $60^{\circ} \mathrm{C}$ for $30 \mathrm{sec}$.

Statistical analysis. In this experiment, the expression of genes in patients with PE and healthy controls of the GSE60438 dataset were analyzed with unpaired Student's t-test using the limma package for R. GO and KEGG pathway analyses were compared using Fisher's exact test. The false discovery rate was controlled within the appropriate range using the Benjamini-Hochberg method.Differences in the mRNA expression of proteasome 26S subunit, non-ATPase 14 (PSMD14), ubiquinol-cytochrome $c$ reductase core protein 2 (UQCRC2) and prostaglandin E synthase 3 (PTGES3) between PE and healthy controls were compared using unpaired Student's t-test. Data were presented as mean $\pm \mathrm{SD}$. $\mathrm{P}<0.05$ was considered to indicate a statistically significant difference. The software used for analysis was GraphPad Prism 7 (GraphPad Software, Inc.).

\section{Results}

Identification of DEGs involved in PE. By performing data normalization and comparison, 441 DEGs were identified in $\mathrm{PE}$ based on the cut-off values $(\mathrm{P}<0.05$ and Ifold-changel $\geq 1.2)$. A volcano plot of DEGs could significantly discriminate between patients with $\mathrm{PE}$ and normotensive pregnant woman (Fig. 1). A heatmap plot showed the clustering of DEGs in PE and normotensive pregnant woman (Fig. 2).

GO BP and KEGG pathway enrichment analysis of the DEGs. The top seven KEGG pathways enriched with DEGs in PE are presented in Fig. 3A. The DEGs were enriched in 'circadian rhythm'. BP terms that were enriched with DEGs were 'protein stabilization', 'positive regulation of telomere maintenance via telomerase,' 'circadian regulation of gene expression' and 'circadian rhythm' (Fig. 3B). 


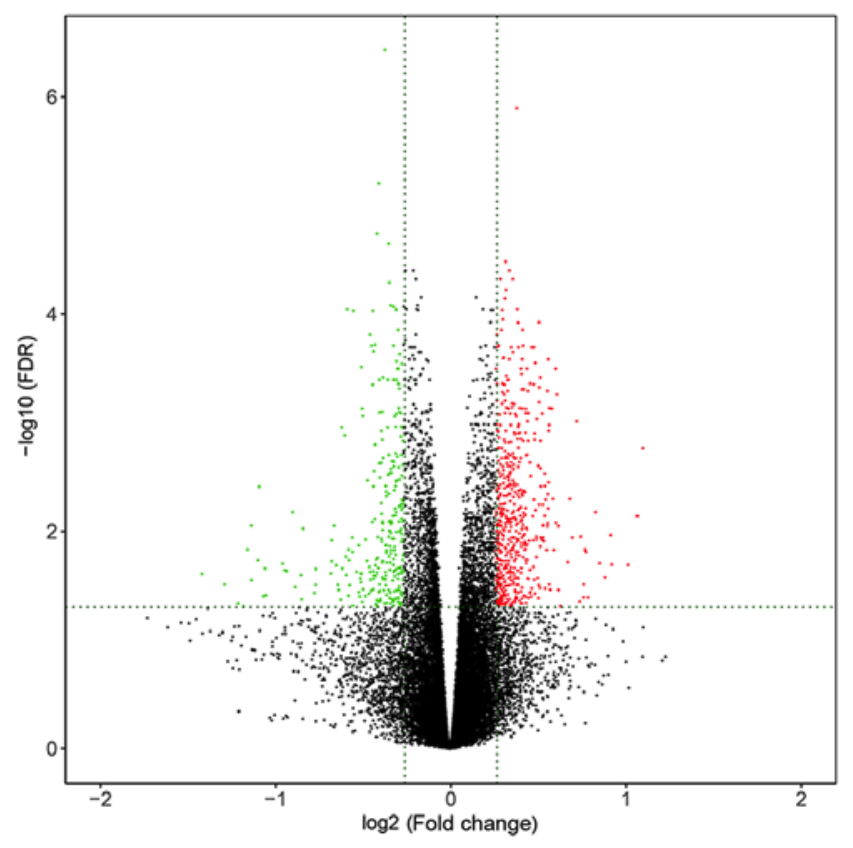

Figure 1. Volcano plot of DEGs. Red, upregulated DEGs with fold-change $\geq 1.2$ and $\mathrm{P}<0.05$. Green, downregulated DEGs with fold-change $\leq-1.2$ and $\mathrm{P}<0.05$. Black, DEGs with fold-change $<1.2$ and $\mathrm{P} \geq 0.05$. DEGs, differentially expressed genes; FDR, false discovery rate.

GSEA in PE. In GO BP analysis of the dataset, 1,706 signaling pathways were upregulated. There were 57 signaling pathways with $\mathrm{P}<0.05$. Of these, four had $\mathrm{P}<0.01$. In KEGG pathway analysis of the dataset, 96 signaling pathways were upregulated. Of these, one had $\mathrm{P}<0.01$ (Fig. 4). KEGG results revealed that DEGs were significantly enriched in 'fatty acid metabolism'. GO results revealed that DEGs were enriched in 'DNA damage response detection of DNA damage', 'regulation of DNA repair', 'positive regulation of small GTPase mediated signal transduction', 'endothelial cell development', 'ERBB2 signaling pathway', 'positive regulation of cytokinesis' and 'cellular response to estradiol stimulus'.

Identification of key genes of PE. The PPI network presented 590 experimentally validated interactions with a combined score $>0.4$. In the present study, the top five genes with higher degrees were screened as hub genes in the PPI network using CytoHubba, including PSMD14, PTGES3, UQCRC2, small ubiquitin like modifier 2 and actin-related protein 3 (Fig. 5). On the basis of the degree of importance, the three most significant modules were screened from the PPI network using MCODE (Fig. 6A, C and E). The KEGG pathway enrichment analysis demonstrated that the most significant modules were primarily associated with 'circadian rhythm', 'glycolysis/gluconeogenesis', 'carbon metabolism' and 'neuroactive ligand-receptor interaction' (Fig. 6B, D and F).

CMAP analysis of the DEGs. The gene expression profiles of MCF7 breast cancer cells, PC3 prostate carcinoma cells and HL60 acute myeloid leukemia cells treated with drugs were divided into positive and negative regulatory gene groups. After eliminating the invalid probes (Iconnectivity scorel $<0.7), 169$ positive regulatory genes and 253 negative regulatory genes were identified. Additionally, drug predictive results similar to and opposite to $\mathrm{PE}$ in gene expression after drug treatment were obtained. According to drug prediction, 20 drugs produced similar gene expression patterns to PE. In Table II, drugs that may aggravate PE are presented. There were also 20 antipsychotic drugs, hypotensor and diuretics identified that may induce gene expression contrary to PE and may have improved effects on the treatment of PE (Table III). Excluding prohibited medicines for pregnant women, furosemide and droperidol were selected based on higher negative scores. The molecular structures and running sums of furosemide and droperidol are shown in Fig. 7. The running sum was based on the genomic expression profile of a drug, with which all the CMAP genomic expression profiles were compared. Finally, the drugs with the highest score of positive association (closest to +1$)$ were considered to be potentially associated with the reference drug at the downstream gene regulation and clinical drug response levels.

Verification of key genes in PE using RT-qPCR. A total of 38 placenta specimens were analyzed, 28 placenta specimens from patients with PE and 10 placenta specimens from healthy pregnant women, to confirm the results of the bioinformatics screening. The primer specificity and the optimal annealing temperatures for five pairs of primers were used for RT-qPCR (Table I). The mRNA expression of five genes were detected by RT-qPCR. The expression levels of PSMD14 and UQCRC2 were confirmed to be lower in the placenta of patients with PE compared with normotensive pregnant woman, while the expression levels of PTGES3 were higher in placentas of patients with PE compared with normotensive pregnant woman (Fig. 8). The expression levels of ACTR3 and SUMO2 in the placenta of patients with PE presented no marked difference compared with normotensive pregnant women (data not shown). These findings indicated that the DEGs may be associated with the pathogenesis of PE.

\section{Discussion}

$\mathrm{PE}$ is a multi-organ disease characterized by hypertension and proteinuria during pregnancy, which is one of the main causes of morbidity and mortality of pregnant women and perinatal infants. At present, the pathological mechanism of PE has not been elucidated. However, studies have indicated that the symptoms and signs of PE are caused by multiple factors, mechanisms and pathways.

Based on GO and KEGG analysis in the current study, a significant portion of DEGs were enriched in the circadian rhythm pathway. However, there are a limited number of studies on the association between alterations of the circadian rhythm and PE (35-37). The circadian rhythm of blood pressure has been explored in relation to the severity and progression of PE (38). Van den Berg et al (39) also indicated that the DNA methylation status of circadian clock and clock-controlled genes in placental tissue was different between patients with $\mathrm{PE}$ and healthy controls.

The KEGG result of GSEA in the current study suggested that DEGs may affect the progression of PE via the fatty acid metabolism pathway. Increased blood lipids and free fatty acids, caused by abnormal lipid and fatty acid metabolism, 

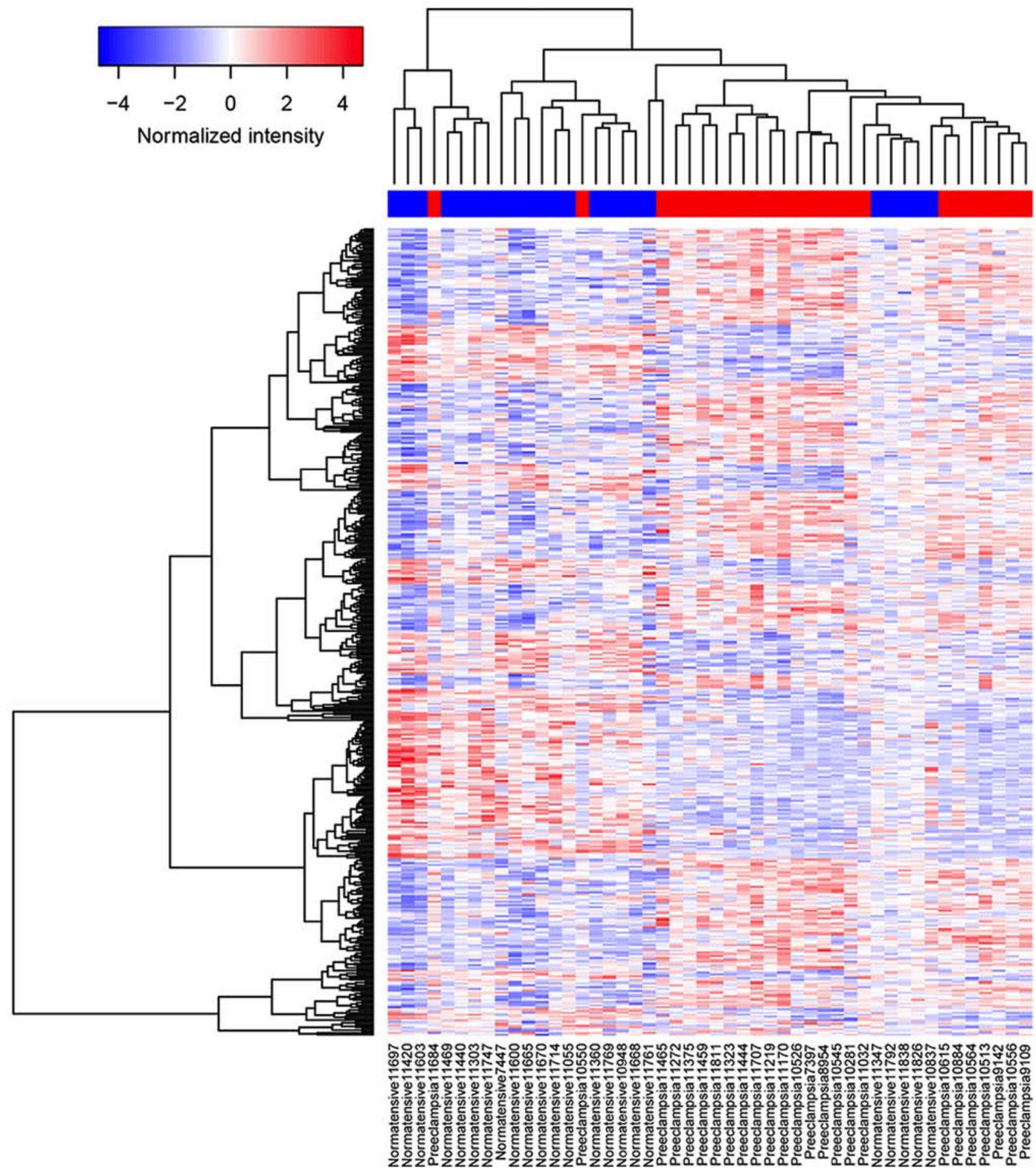

Figure 2. Heatmap plot of differentially expressed genes between patients with preeclampsia and healthy pregnant women. Red, upregulated genes and blue, downregulated genes.

could enhance oxidative stress, inflammation, endothelial dysfunction and promote the occurrence of PE (40). The GO results of GSEA in the present study indicated that the incidence of PE was related to 'DNA damage response detection of DNA damage', 'regulation of DNA repair' and 'endothelial cell development', which is closely related to oxidative stress. Small artery spasms, low perfusion of organs and tissue hypoxia during PE have been reported to lead to excessive production of oxygen free radicals, which can result in protein oxidation, DNA mutation and breakage, and endothelial cell damage (41-44). According to the results of the present study, positive regulation of GTPase-mediated signal transduction may serve a key role in the occurrence of PE. The Rho family of GTPases are small molecule guanosine-binding proteins. Activation of the Rho/Rho-associated coiled-coil containing protein kinase (ROCK) pathway can lead to injury of vascular endothelial cells, small vessel spasms and eventually PE symptoms (45). In addition, according to the present study the 

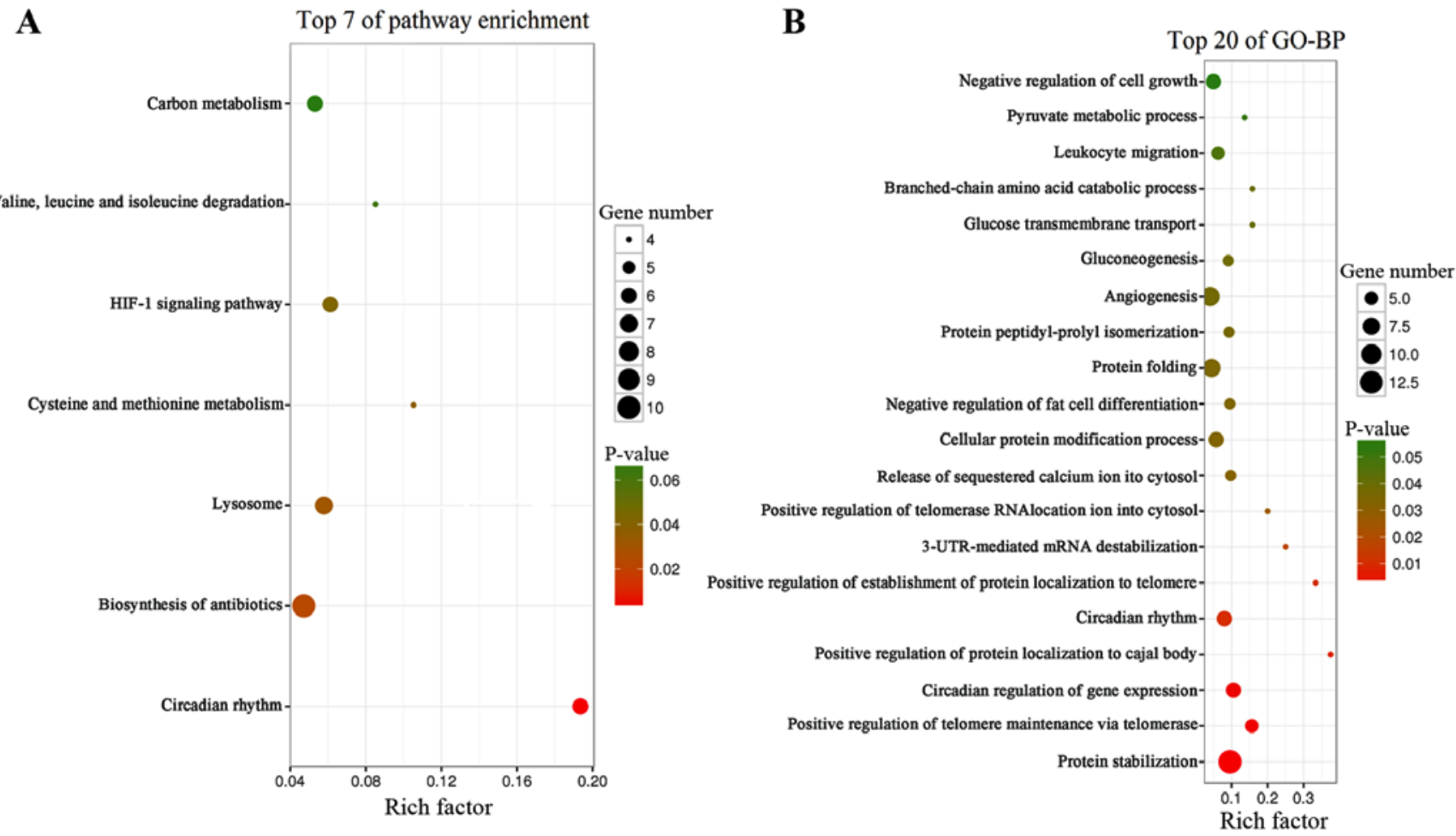

Figure 3. Functional enrichment analyses of the differentially expressed genes. (A) Results of Kyoto Encyclopedia of Genes and Genomes pathway analysis (B) Analysis results of GO BP. GO, Gene Ontology; BP, biological process.

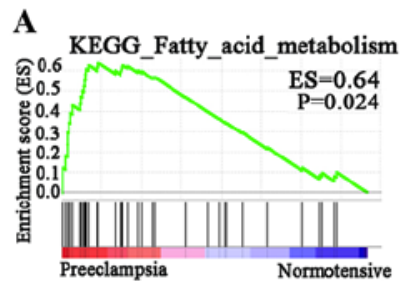

E

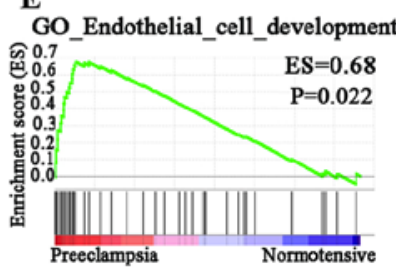

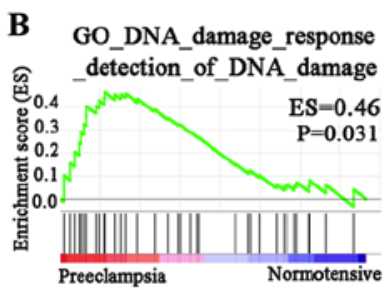

$\mathbf{F}$

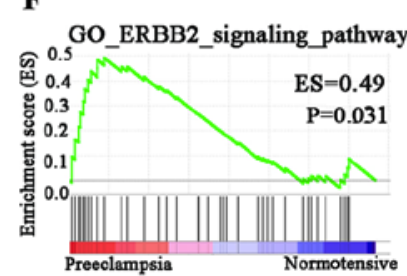

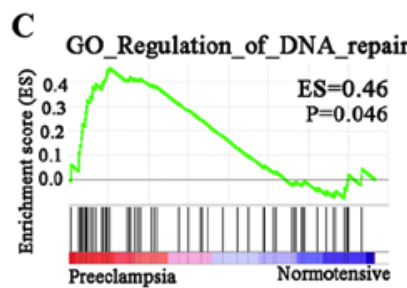

G

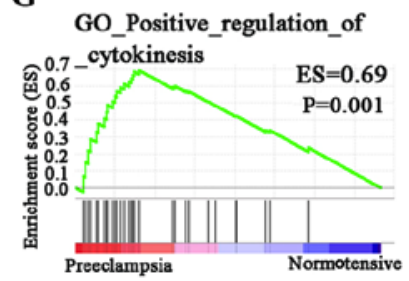

D GO_Positive_regulation_of_small_ GTPase_mediated_signal_transduction

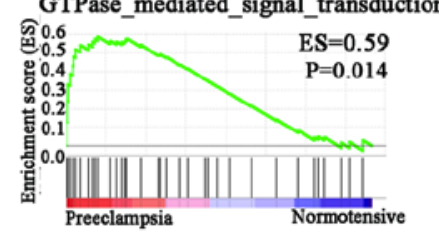

$\mathbf{H}$

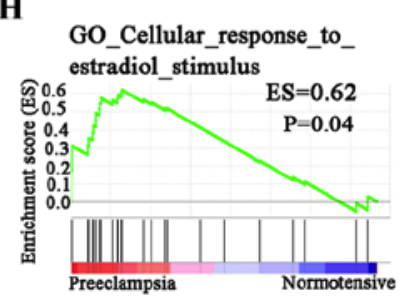

Figure 4. Significant enrichment plots in preeclampsia using GSEA. GSEA results showed enrichent in (A) 'Fatty acid metabolism', (B) 'DNA damage response detection of DNA damage', (C) 'Regulation of DNA repair', (D) 'Positive regulation of small GTPase mediated signal transduction', (E) 'Endothelial cell development', (F) 'ERBB2 signaling pathway', (G) 'Positive regulation of cytokinesis' and (H) 'Cellular response to estradiol stimulus'. GSEA, gene set enrichment analysis; GO, Gene Ontology.

occurrence of PE may be associated with the Erb-B2 receptor tyrosine kinase 2 signaling pathway, positive regulation of cytokines and cellular response to estradiol stimulus.

In the present study, CMAP analysis revealed the association between PE and certain drugs. Furosemide and droperidol induced gene expression that was contrary to PE. Furosemide is a diuretic that is used to treat eclampsia complicated with intracranial hypertension (46). Droperidol has strong antipsychotic effects that are mainly related to its antagonism to dopamine receptors; it promotes the transformation of dopamine in the brain (47). These two drugs may prove to have therapeutic benefits in preventing the development or progression of PE.

Through the construction of a PPI network, five hub genes with higher degrees were identified. However, only PSMD14, PTGES3 and UQCRC2 were verified to be significantly different between placenta samples from patients with PE and normotensive pregnant woman. PSMD14 is one of the 19 essential subunits of a completely assembled 19S proteasome complex (48). In the current study, the expression of PSMD14 was significantly lower in the placenta of patients with PE compared with normotensive pregnant 


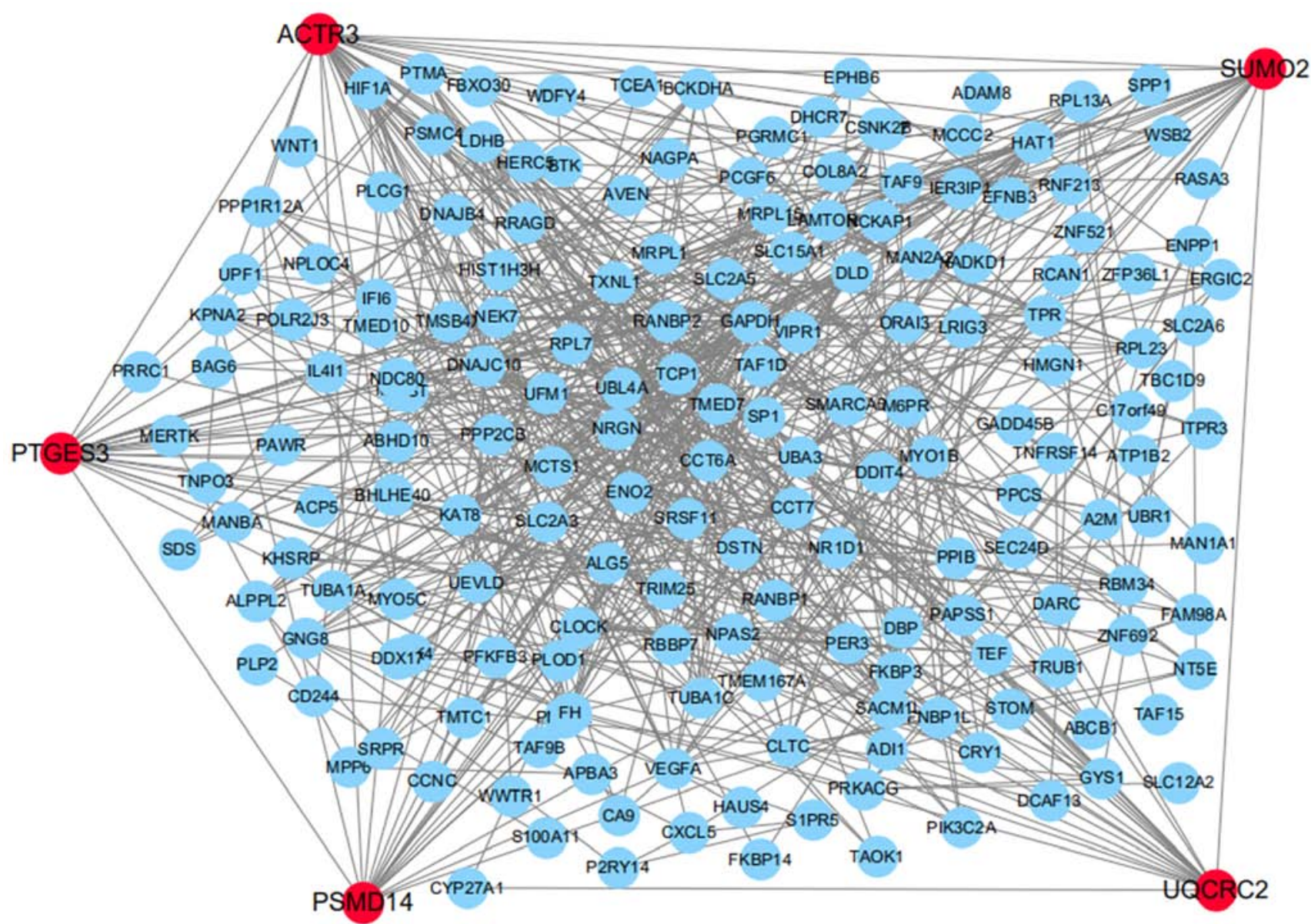

Figure 5. Protein-protein interaction network of differentially expressed genes in placenta samples between patients with preeclampsia and healthy pregnant women. Red dots are indicative of hub genes and bule dots are indicative of non-hub genes.

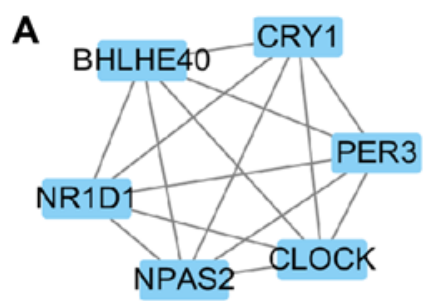

C

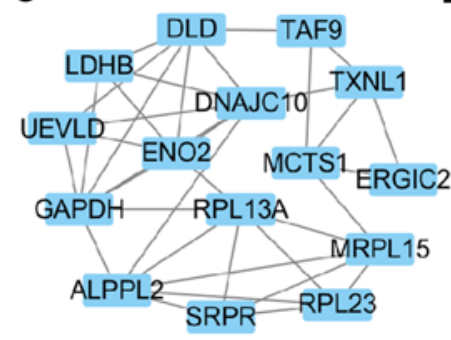

E

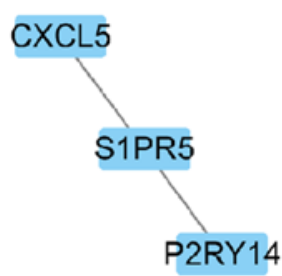

B

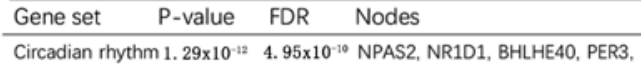

CRY1, CLOCK

\begin{tabular}{lccl}
\hline Gene set & P-value & FDR & Nodes \\
\hline Glycolysis/Gluconeogenesis & $7.02 \times 10^{-5}$ & 0.05 & LDHB, DLD, ENO2 \\
\hline Biosynthesis of antibiotics & 0.002 & 1.57 & LDHB, DLD, ENO2 \\
\hline Carbon metabolism & 0.008 & 6.54 & DLD, ENO2 \\
\hline Ribosome & 0.012 & 9.23 & MRPL15, RPL23, RPL13A \\
\hline
\end{tabular}

Ribosome $\quad 0.012$

\begin{tabular}{lccc} 
& & & \\
\hline Gene set & P-value & FDR & Nodes \\
\hline Neuroactive ligand-receptor interaction & 0.07 & 29.94 & P2RY14, S1PR5 \\
\hline
\end{tabular}

Figure 6. Screening of top three modules using MCODE. (A, C and E) Top three modules (highly interconnected regions) screened from the protein-protein interaction network using MCODE and (B, D and F) pathways involved in each module. MCODE, Molecular Complex Detection; FDR, false discovery rate. 
Table II. Drug prediction results similar to preeclampsia in gene expression after drug treatment.

\begin{tabular}{rrlclclcc}
\hline Rank & Batch & CMAPname & Dose $(\mu \mathrm{M})$ & Cell & Score & Up & Down & Instance_ID \\
\hline 1 & 694 & Nystatin & 4 & MCF7 & 1 & 0.236 & -0.254 & 4807 \\
2 & 1,068 & 0198306-0000 & 10 & MCF7 & 0.922 & 0.193 & -0.259 & 7064 \\
3 & 743 & Nocodazole & 13 & MCF7 & 0.919 & 0.212 & -0.239 & 6793 \\
4 & 693 & R-atenolol & 15 & PC3 & 0.894 & 0.17 & -0.268 & 4259 \\
5 & 695 & Cyclopenthiazide & 11 & MCF7 & 0.879 & 0.183 & -0.248 & 4813 \\
6 & 704 & Sulpiride & 12 & PC3 & 0.865 & 0.143 & -0.281 & 4566 \\
7 & 756 & Nomegestrol & 11 & MCF7 & 0.846 & 0.196 & -0.219 & 6525 \\
8 & 745 & Dizocilpine & 12 & MCF7 & 0.844 & 0.153 & -0.261 & 6223 \\
9 & 1,082 & Irinotecan & 100 & MCF7 & 0.843 & 0.191 & -0.222 & 7498 \\
10 & 744 & Clobetasol & 9 & MCF7 & 0.84 & 0.198 & -0.214 & 6835 \\
11 & 702 & Eucatropine & 12 & PC3 & 0.838 & 0.171 & -0.24 & 4316 \\
12 & 693 & Picrotoxinin & 14 & PC3 & 0.836 & 0.182 & -0.229 & 4260 \\
13 & 695 & Simvastatin & 10 & MCF7 & 0.828 & 0.158 & -0.248 & 4828 \\
14 & 701 & Phenformin & 17 & PC3 & 0.823 & 0.19 & -0.213 & 4283 \\
15 & 702 & Promazine & 12 & PC3 & 0.814 & 0.157 & -0.243 & 4308 \\
16 & 694 & Benperidol & 10 & MCF7 & 0.813 & 0.13 & -0.269 & 4781 \\
17 & 718 & Allantoin & 25 & PC3 & 0.807 & 0.175 & -0.221 & 5052 \\
18 & 693 & Sulfaguanidine & 19 & PC3 & 0.806 & 0.213 & -0.183 & 4257 \\
19 & 694 & Mestranol & 13 & MCF7 & 0.801 & 0.126 & -0.267 & 4792 \\
20 & 756 & Alfaxalone & 12 & MCF7 & 0.801 & 0.172 & -0.22 & 6514 \\
\hline
\end{tabular}

Table III. Drug prediction results opposite to preeclampsia in gene expression after drug treatment.

\begin{tabular}{rrlclllll}
\hline Rank & Batch & CMAP name & Dose $(\mu \mathrm{M})$ & Cell & Score & Up & Down & Instance_ID \\
\hline 1 & 731 & Tetrahydroalstonine & 11 & PC3 & -0.763 & -0.135 & 0.246 & 5728 \\
2 & 694 & Zardaverine & 15 & MCF7 & -0.768 & -0.137 & 0.246 & 4793 \\
3 & 692 & Idoxuridine & 11 & PC3 & -0.769 & -0.168 & 0.216 & 4200 \\
4 & 1,059 & PF-00562151-00 & 10 & MCF7 & -0.773 & -0.154 & 0.232 & 6912 \\
5 & 694 & Pyrimethamine & 16 & MCF7 & -0.781 & -0.14 & 0.249 & 4779 \\
6 & 692 & Epitiostanol & 13 & PC3 & -0.782 & -0.141 & 0.248 & 4204 \\
7 & 706 & Prestwick-984 & 9 & MCF7 & -0.782 & -0.174 & 0.216 & 4948 \\
8 & 706 & Retrorsine & 11 & MCF7 & -0.783 & -0.175 & 0.215 & 4946 \\
9 & 694 & Gabexate & 10 & MCF7 & -0.784 & -0.176 & 0.215 & 4804 \\
10 & 692 & Naftopidil & 9 & PC3 & -0.788 & -0.165 & 0.228 & 4193 \\
11 & 1,059 & SB-202190 & 1 & MCF7 & -0.791 & -0.145 & 0.25 & 6909 \\
12 & 630 & Droperidol & 11 & HL60 & -0.791 & -0.221 & 0.173 & 1290 \\
13 & 690 & Boldine & 12 & MCF7 & -0.807 & -0.165 & 0.238 & 4122 \\
14 & 694 & Zalcitabine & 19 & MCF7 & -0.817 & -0.161 & 0.247 & 4799 \\
15 & 677 & Iopanoic acid & 7 & MCF7 & -0.818 & -0.174 & 0.234 & 3527 \\
16 & 692 & Molindone & 13 & PC3 & -0.838 & -0.192 & 0.226 & 4199 \\
17 & 693 & Amprolium & 13 & PC3 & -0.855 & -0.193 & 0.233 & 4241 \\
18 & 702 & 0317956-0000 & 10 & PC3 & -0.859 & -0.222 & 0.206 & 4331 \\
19 & 728 & Furosemide & 12 & PC3 & -0.86 & -0.162 & 0.267 & 4503 \\
20 & 694 & Meptazinol & 15 & MCF7 & -1 & -0.245 & 0.254 & 4774 \\
\hline
\end{tabular}

woman. Therefore, the activity and function of the proteasome may be decreased in patients with PE. A previous study demonstrated that proteasomal activity and function was decreased by $\sim 30 \%$ in PE placenta (49). Proteasomes degrade oxidative stress proteins that destroy cells and tissues. Previous studies have shown that the oxidative 
Furosemide

$\left(\mathrm{C}_{12} \mathrm{H}_{11} \mathrm{CIN}_{2} \mathrm{O}_{5} \mathrm{~S}\right)$
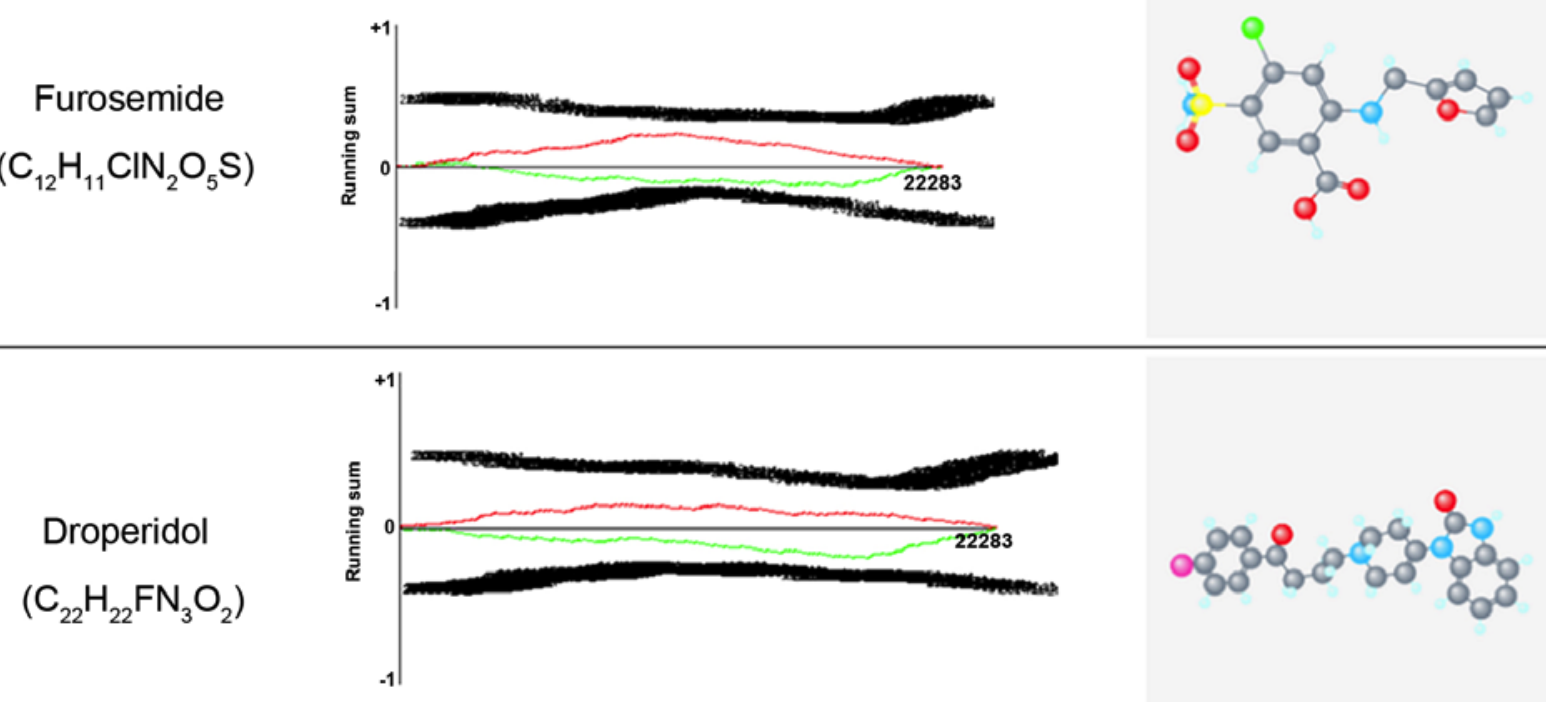

Figure 7. A graphical view of running sum and molecular structure for furosemide and droperidol. CMAP, connectivity map.
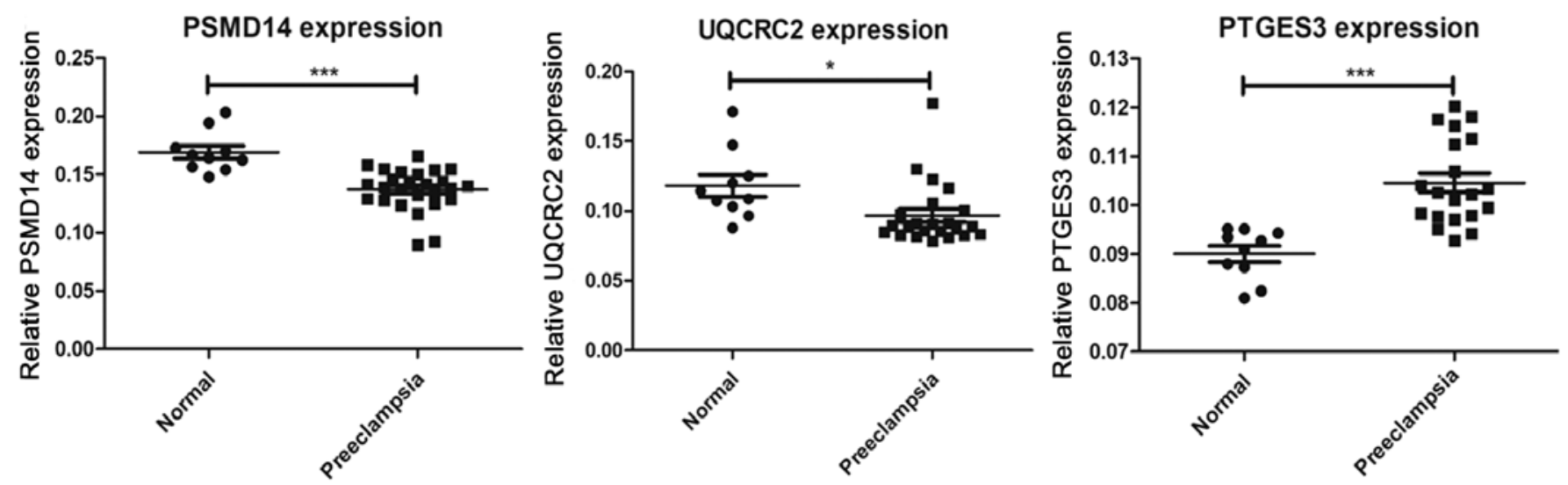

Figure 8. Three key genes that show significantly different expression levels in placenta samples from patients with $\mathrm{PE}$ and normal healthy controls. "P<0.05; ${ }^{* * * *} \mathrm{P}<0.001$. PSMD14, proteasome 26S subunit, non-ATPase 14; PTGES3, prostaglandin E synthase 3; UQCRC2, ubiquinol-cytochrome $c$ reductase core protein 2.

stress-associated proteins were increased by $\sim 30 \%$ in the placenta of patients with PE compared with the placenta of healthy pregnant women (50-52). Oxidative stress-associated proteins mainly refer to the products of oxidative stress, such as damaged DNA bases, protein oxidation products and lipid peroxidation products. These findings suggested that decreased proteasomal activity due to reduced expression of PSMD14 could lead to the accumulation of oxidatively damaged proteins in PE.

UQCRC2, a member of the peptidase M16 family, is an indispensable component of mitochondrial complex III, which is a part of the mitochondrial respiratory chain $(53,54)$. In the present study, the expression of UQCRC2 was lower in placenta samples from patients with PE compared with in healthy placenta samples. According to a previous study,
UQCRC2 may be involved in the production of intracellular reactive oxygen species (ROS) and oxidative stress (53). In addition, decreased expression of UQCRC2 led to increased cellular ROS level (53). PTGES3 is a molecular chaperone located in genomic response elements that may break down transcriptional regulatory complexes and destroy receptor-mediated transcriptional activation (55). PTGES3 is essential for the normal physiological function of the glucocorticoid receptor and other steroid receptors. Disordered steroid synthesis and metabolism is an important factor leading to PE (56). PSMD14, PTGES3 and UQCRC2 are all involved in oxidative stress, carcinoma, and steroid synthesis and metabolism (57-59). The changes in mRNA expression of PSMD14, PTGES3 and UQCRC2 detected in the present study suggested that carcinoma and oxidative 
stress signals may be involved in the occurrence and development of PE. In the current study, patients with PE exhibited decreased expression of PSMD14 and UQCRC2. Previous studies have shown that PSMD14 and UQCRC2 are involved in oxidative stress signals; decreased expression of PSMD14 and UQCRC2 is associated with oxidative damage to vascular endothelial cells $(49,53)$. In the present study, PTGES3 exhibited increased expression in patients with PE. It has been reported that PTGES3 may be involved in steroid synthesis and metabolism signals; abnormal expression of PTGES3 can lead to disordered steroid synthesis and metabolism, and thus to the development of PE (56). In addition, it has been reported that UQCRC2 is involved in carcinoma cell signaling; it is of note that carcinoma signaling pathways, such as Notch, RhoA/ROCK and NF- $\kappa B$, are also related to PE (60-62). Abnormal expression of UQCRC2 can result in disorders of carcinoma signaling and also possibly the occurrence of PE.

In conclusion, the pathogenesis of PE has been found to involve multiple cellular signaling pathways and BPs. The present study indicated that furosemide and droperidol may have beneficial effects as PE treatments. Furthermore, the downregulation of PSMD14 and UQCRC2 expression may induce increased oxidative stress in PE, whereas the upregulation of PTGES3 expression may lead to disordered steroid synthesis and metabolism, which could participate in the development of PE. Therefore, these three hub genes may be biomarkers or therapeutic targets in PE.

\section{Acknowledgements}

Not applicable.

\section{Funding}

The present study was supported by the Chinese National Natural Science Foundation Grant (grant nos. 81671541, 81273202 and 81701545), the Special Program for Key Technology of the Health and Family Planning of Zhenjiang City, Jiangsu, P.R. China (grant no. SHW2017003), Clinical Medicine Science \& Technology Project of Jiangsu province of China (grant no. BL2013024), Key Research and Development Programs Social Development Project of Science and Technology Commission Foundation of Jiangsu Province (grant no. BE2016721), Jiangsu Undergraduate Training Program for Innovation \& Entrepreneurship (grant no. 201710299063Y) and Advance Research Fund Project of The Second Affiliated Hospital of Soochow University (grant no. SDFEYQN1910).

\section{Availability of data and materials}

The datasets used and/or analyzed during the current study are available from the corresponding author on reasonable request. The dataset GSE60438 is available from https://www. ncbi.nlm.nih.gov/geo/query/acc.cgi?acc=GSE60438.

\section{Authors' contributions}

$\mathrm{ZX}$ and $\mathrm{CW}$ performed the experiment and wrote the manuscript. YL and NW analyzed and interpreted the patient data regarding preeclampsia. SG, SQ, ZW, JD, LZ, HW and WW collected the placenta of patients with PE and normotensive pregnant woman. BW, JY, JF and PY performed the bioinformatics analyses. NW and QS funded the experiment and designed this research. All authors read and approved the final version of the manuscript.

\section{Ethics approval and consent to participate}

The researchers obtained verbal informed consent before collecting patient samples. Written informed consent was waived by the Ethics Committee of the Affiliated Hospital of Jiangsu University. The experiment was approved by the Ethics Committee of the Affiliated Hospital of Jiangsu University (approval no. 20170415).

\section{Patient consent for publication}

Not applicable.

\section{Competing interests}

The authors declare that they have no competing interests.

\section{References}

1. Sánchez-Aranguren LC, Prada CE, Riaño-Medina CE and Lopez M: Endothelial dysfunction and preeclampsia: Role of oxidative stress. Front Physiol 5: 372, 2014.

2. Wang Y, Zhang X, Cheng GM and Ren CC: Expression of transforming growth factor-beta 1, vascular cell adhesion molecule 1 and E-selectin in placenta of patients with pre-clampsia. Zhonghua Fu Chan Ke Za Zhi 41: 514-517, 2006 (In Chinese).

3. Weissgerber TL and Mudd LM: Preeclampsia and diabetes. Curr Diab Rep 15: 9, 2015.

4. Spradley FT: Metabolic abnormalities and obesity's impact on the risk for developing preeclampsia. Am J Physiol Regul Integr Comp Physiol 312: R5-R12, 2017.

5. Karumanchi SA and Granger JP: Preeclampsia and pregnancy-related hypertensive disorders. Hypertension 67: 238-242, 2016.

6. Facca TA, Kirsztajn GM and Sass N: Preeclampsia (marker of chronic kidney disease): From genesis to future risks. J Bras Nefrol 34: 87-93, 2012 (In English, Portuguese).

7. Kumasawa K, Ikawa M, Kidoya H, Hasuwa H, Saito-Fujita T, Morioka Y, Takakura N, Kimura T and Okabe M: Pravastatin induces placental growth factor (PGF) and ameliorates preeclampsia in a mouse model. Proc Natl Acad Sci USA 108: 1451-1455, 2011.

8. Filipek A and Jurewicz E: Preeclampsia - a disease of pregnant women. Postepy Biochem 64: 232-229, 2018.

9. Jiang $J$ and Zhao ZM: LncRNA HOXD-AS1 promotes preeclampsia progression via MAPK pathway. Eur Rev Med Pharmacol Sci 22: 8561-8568, 2018.

10. Zhang XM, Xiong X, Tong C, Li Q, Huang S, Li QS, Liu YM, Li HY, Baker P, Shan N and Qi HB: Down-regulation of laminin (LN)- $\alpha 5$ is associated with preeclampsia and impairs trophoblast cell viability and invasiveness through PI3K signaling pathway. Cell Physiol Biochem 51: 2030-2040, 2018.

11. Pan Q, Shai O, Lee LJ, Frey BJ and Blencowe BJ: Deep surveying of alternative splicing complexity in the human transcriptome by high-throughput sequencing. Nat Genet 40: 1413-1415, 2008.

12. Luo S, Cao N, Tang Y and Gu W: Identification of key microRNAs and genes in preeclampsia by bioinformatics analysis. PLoS One 12: e0178549, 2017.

13. Zhu X, Yang Y,Han T, Yin G, Gao P, Ni Y, Su X, Liu Y and Yao Y: Suppression of microRNA-18a expression inhibits invasion and promotes apoptosis of human trophoblast cells by targeting the estrogen receptor alpha gene. Mol Med Rep 12: 2701-2706, 2015.

14. Barrett T, Troup DB, Wilhite SE, Ledoux P, Rudnev D, Evangelista C, Kim IF, Soboleva A, Tomashevsky M, Marshall KA, et al: NCBI GEO: Archive for high-throughput functional genomic data. Nucleic Acids Res 37 (Database Issue): D885-D890, 2009. 
15. Subramanian A, Tamayo P, Mootha VK, Mukherjee S, Ebert BL Gillette MA, Paulovich A, Pomeroy SL, Golub TR, Lander ES and Mesirov JP: Gene set enrichment analysis: A knowledge-based approach for interpreting genome-wide expression profiles. Proc Natl Acad Sci USA 102: 15545-15550, 2005.

16. Lamb J, Crawford ED, Peck D, Modell JW, Blat IC, Wrobel MJ, Lerner J, Brunet JP, Subramanian A, Ross KN, et al: The connectivitiy map: Using gene-expression signatures to connect small molecules, genes, and disease. Scinece 313: 1929-1935, 2006

17. Huang HL, Peng CY, Lai MJ, Chen CH, Lee HY, Wang JC, Liou JP, Pan SL and Teng CM: Novel oral histone deacetylase inhibitor, MPT0E028, displays potent growth-inhibitory activity against human B-cell lymphoma in vitro and in vivo. Oncotarget 6: 4976-4991, 2015.

18. Wen Z, Wang Z, Wang S, Ravula R, Yang L, Xu J, Wang C, Zuo Z, Chow MS, Shi L and Huang Y: Discovery of molecular mechanisms of traditional Chinese medicinal formula Si-Wu-Tang using gene expression microarray and connectivity map. PLOS One 6: e18278, 2011.

19. Wei LL, Pan YS, Tang Q, Yang ZJ, Song WQ, Gao YF, Li J, Zhang L and Liu SG: Decreased ALCAM expression and promoter hypermethylation is associated with preeclampsia. Hypertens Res 43: 13-22, 2020.

20. Wang Q, Lu X, Li C, Zhang W, Lv Y, Wang L, Wu L, Meng L, Fan Y, Ding $\mathrm{H}$, et al: Down-regulated long non-coding RNA PVT1 contributes to gestational diabetes mellitus and preeclampsia via regulation of human trophoblast cells. Biomed Pharmacother 120: 109501, 2019.

21. Hu S, Li J, Tong M, Li Q, Chen Y, Lu H, Wang Y and Min L: MicroRNA-144-3p may participate in the pathogenesis of preeclampsia by targeting Cox-2. Mol Med Rep 19: 4655-4662, 2019.

22. Dong K, Zhang X, Ma L, Gao N, Tang H, Jian F and Ma Y: Downregulations of circulating miR-31 and miR-21 are associated with preeclampsia. Pregnancy Hypertens 17: 59-63, 2019.

23. Wang Y, Cheng K, Zhou W, Liu H, Yang T, Hou P and Li X miR-141-5p regulate ATF2 via effecting MAPK1/ERK2 signaling to promote preeclampsia. Biomed Pharmacother 115: 108953, 2019.

24. Yong HE, Melton PE, Johnson MP, Freed KA, Kalionis B, Murthi P, Brennecke SP, Keogh RJ and Moses EK: Genome-wide transcriptome directed pathway analysis of maternal pre-eclampsia susceptibility genes. PLoS One 10: e0128230, 2015.

25. Cao C, Chen J, Lyu C, Yu J, Zhao W, Wang Y and Zou D: Bioinformatics analysis of the effects of tobacco smoke on gene expression. PLoS One 10: e0143377, 2015.

26. Ashburner M, Ball CA, Blake JA, Botstein D, Butler $\mathrm{H}$, Cherry JM, Davis AP, Dolinski K, Dwight SS, Eppig JT, et al: Gene ontology: Tool for the unification of biology. The gene ontology consortium. Nat Genet 25: 25-29, 2000.

27. Kanehisa M and Goto S: KEGG: Kyoto encyclopedia of genes and genomes. Nucleic Acids Res 28: 27-30, 2000.

28. Huang da W, Sherman BT and Lempicki RA: Systematic and integrative analysis of large gene lists using DAVID bioinformatics resources. Nat Protoc 4: 44-57, 2009.

29. Mootha VK, Lindgren CM, Eriksson KF, Subramanian A, Sihag S, Lehar J, Puigserver P, Carlsson E, Ridderstråle M, Laurila E, et al: PGC-1alpha-responsive genes involved in oxidative phosphorylation are coordinately downregulated in human diabetes. Nat Genet 34: 267-273, 2003.

30. Chin CH, Chen SH, Wu HH, Ho CW, Ko MT and Lin CY: cytoHubba: Identifying hub objects and sub-networks from complex interactome. BMC Syst Biol 4 (Suppl 4): S11, 2014.

31. Bader GD and Hogue CW: An automated method for finding molecular complexes in large protein interaction networks. BMC Bioinformatics 4: 2, 2003.

32. Lamb J, Crawford ED, Peck D, Modell JW, Blat IC, Wrobel MJ, Lerner J, Brunet JP, Subramanian A, Ross KN, et al: The connectivity map: Using gene-expression signatures to connect small molecules, genes, and disease. Science 313: 1929-1935, 2006.

33. Group of hypertensive disorders of pregnancy, branch of Obstetrics and Gynecology, Chinese Medical Association: Chin J Obst Gynecol 10, 2015.

34. Livak KJ and Schmittgen TD: Analysis of relative gene expression data using real-time quantitative PCR and the 2(-Delta Delta C(T)) method. Methods 25: 402-408, 2001

35. Ditisheim AJ, Dibner C, Philippe $\mathrm{J}$ and Pechère-Bertschi A Biological rhythms and preeclampsia. Front Endocrinol (Lausanne) 4: 47, 2013
36. Haelterman E, Marcoux S, Croteau A and Dramaix M: Population-based study on occupational risk factors for preeclampsia and gestational hypertension. Scand J Work Environ Health 33: 304-317, 2007.

37. Mozurkewich EL, Luke B, Avni M and Wolf FM: Working conditions and adverse pregnancy outcome: A meta-analysis. Obstet Gynecol 95: 623-635, 2000.

38. Munz W, Seufert R, Steiner E, Pollow K and Brockerhoff P: Circadian blood pressure rhythm in preeclampsia as a predictor of maternal and obstetrical outcome. Z Geburtshilfe Neonatol 207: 132-136, 2003 (In German).

39. van den Berg CB, Chaves I, Herzog EM, Willemsen SP, van der Horst GTJ and Steegers-Theunissen RPM: Early- and late-onset preeclampsia and the DNA methylation of circadian clock and clock-controlled genes in placental and newborn tissues. Chronobiol Int 34: 921-932, 2017.

40. Ding X, Yang Z, Han Y and Yu H: Fatty acid oxidation changes and the correlation with oxidative stress in different preeclampsia-like mouse models. PLoS One 9: e109554, 2014.

41. Wata S, Lee JW, Okada K, Lee JK, Iwata M, Rasmussen B, Link TA, Ramaswamy S and Jap BK: Complete structure of the 11-subunit bovine mitochondrial cytochrome bcl complex. Science 281: 64-71, 1998 .

42. Aouache R, Biquard L, Vaiman D and Miralles F: Oxidative stress in preeclampsia and placental diseases. Int J Mol Sci 19: pii: E1496, 2018.

43. Elliot MG: Oxidative stress and the evolutionary origins of preeclampsia. J Reprod Immunol 114: 75-80, 2016.

44. Chiarello DI, Abad C, Rojas D, Toledo F, Vázquez CM, Mate A, Sobrevia L and Marín R: Oxidative stress: Normal pregnancy versus preeclampsia. Biochim Biophys Acta Mol Basis Dis 1866: 165354,2020

45. Heneweer C, Kruse LH, Kindhauser F, Schmidt M, Jakobs KH, Denker HW and Thie M: Adhesiveness of human uterine epithelial RL95-2 cells to trophoblast: Rho protein regulation. Mol Hum Reprod 8: 1014-1022, 2002.

46. Tamás P, Hantosi E, Farkas B, Ifi Z, Betlehem J and Bódis J: Preliminary study of the effects of furosemide on blood pressure during late-onset pre-eclampsia in patients with high cardiac output. Int J Gynaecol Obstet 136: 87-90, 2017.

47. Adamantidis MM, Kerram P, Caron JF and Dupuis BA: Droperidol exerts dual effects on repolarization and induces early afterdepolarizations and triggered activity in rabbit Purkinje fibers. J Pharmacol Exp Ther 266: 884-893, 1993.

48. Gu ZC and Enenkel C: Proteasome assembly. Cell Mol Life Sci 71: 4729-4745, 2014.

49. Hass R and Sohn C: Increased oxidative stress in pre-eclamptic placenta is associated with altered proteasome activity and protein patterns. Placenta 24: 979-984, 2003.

50. Zhou JF, Wang XY, Shangguan XJ, Gao ZM, Zhang SM, Xiao WQ and Chen CG: Increased oxidative stress in women with pregnancy-induced hypertension. Biomed Environ Sci 18: 419-426, 2005 .

51. Mistry HD, Wilso V, Ramsay MM, Symonds ME and Broughton Pipkin F: Reduced selenium concentrations and glutathione peroxidase activity in preeclamptic pregnancies. Hypertension 52: 881-888, 2008.

52. Vanderlelie J, Venardos K, Clifton VL, Gude NM, Clarke FM and Perkins AV: Increased biological oxidation and reduced anti-oxidant enzyme activity in pre-eclamptic placenta. Placenta 26: 53-58, 2005.

53. Miyake N, Yano S, Sakai C, Hatakeyama H, Matsushima Y, Shiina M, Watanabe Y, Bartley J, Abdenur JE, Wang RY, et al: Mitochondrial complex III deficiency caused by a homozygous UQCRC2 mutation presenting with neonatal-onset recurrent metabolic decompensation. Hum Mutat 34: 446-452, 2013.

54. Gaignard P, Eyer D, Lebigot E, Oliveira C, Therond P, Boutron A and Slama A: UQCRC2 mutation in a patient with mitochondrial complex III deficiency causing recurrent liver failure, lactic acidosis and hypoglycemia. J Hum Genet 62: 729-731, 2017.

55. Freeman BC and Yamamoto KR: Disassembly of transcriptional regulatory complexes by molecular chaperones. Science 296 : 2232-2235, 2002

56. Sinclair D, Fillman SG, Webster MJ, and Weickert CS: Dysregulation of glucocorticoid receptor co-factors FKBP5, BAG1 and PTGES3 in prefrontal cortex in psychotic illness. Sci Rep 3: 3539, 2013. 
57. Lv J, Zhang S, Wu H, Lu J, Lu Y, Wang F, Zhao W, Zhan P, Lu J, Fang Q, et al: Deubiquitinase PSMD14 enhances hepatocellular carcinoma growth and metastasis by stabilizing GRB2. Cancer Lett 469: 22-34, 2020

58. Song B, Du J, Feng Y, Gao YJ and Zhao JS: Co-expressed differentially expressed genes and long non-coding RNAs involved in the celecoxib treatment of gastric cancer: An RNA sequencing analysis. Exp Ther Med 12: 2455-2468, 2016.

59. Gu Y, Chen G and Du Y: Screening of prognosis-related genes in primary breast carcinoma using genomic expression data. J Comput Biol, Nov 13, 2019 (Epub ahead of print).

60. Cao W, Wang X, Chen T, Zhu H, Xu W, Zhao S, Cheng X and Xia L: The expression of Notch/Notch ligand, IL-35, IL-17, and Th17/Treg in preeclampsia. Dis Markers 2015: 316182, 2015.
61. Gu Y, Feng Y, Yu J, Yuan H, Yin Y, Ding J, Zhao J, Xu Y, $\mathrm{Xu} \mathrm{J}$ and Che $\mathrm{H}$ : Fasudil attenuates soluble fms-like tyrosine kinase-1 (sFlt-1)-induced hypertension in pregnant mice through RhoA/ROCK pathway. Oncotarget 8: 104104-104112, 2017.

62. Zheng L, Shi L, Zhou Z, Chen X, Wang L, Lu Z and Tang R: Placental expression of AChE, $\alpha 7 n A C h R$ and NF- $\kappa$ B in patients with preeclampsia. Ginekol Pol 89: 249-255, 2018.

(i) (9) This work is licensed under a Creative Commons Attribution-NonCommercial-NoDerivatives 4.0 International (CC BY-NC-ND 4.0) License. 